\title{
The role of intrinsically photosensitive retinal ganglion cells in nonimage-forming responses to light
}

This article was published in the following Dove Press journal:

Eye and Brain

5 September 2012

Number of times this article has been viewed

\section{Daniel M Warthen ${ }^{1,2}$ \\ Ignacio Provencio'}

'Department of Biology, University of Virginia, Charlottesville, VA, USA; 2Department of Pharmacology, University of Virginia School of Medicine, Charlottesville, VA, USA
Correspondence: Ignacio Provencio Department of Biology, University of Virginia, Physical Life Sciences Building, Room 406, 90 Geldard Drive, Charlottesville, VA 22903, USA

$\mathrm{Tel}+$ I 4349244412

Email ip7m@virginia.edu
Abstract: Light exerts many effects on behavior and physiology. These effects can be characterized as either image-forming or nonimage-forming (NIF) visual processes. Image-forming vision refers to the process of detecting objects and organisms in the environment and distinguishing their physical characteristics, such as size, shape, and direction of motion. NIF vision, in contrast, refers to effects of light that are independent of fine spatiotemporal vision. NIF effects are many and varied, ranging from modulation of basal physiology, such as heart rate and body temperature, to changes in higher functions, such as mood and cognitive performance. In mammals, many NIF effects of light are dependent upon the inner retinal photopigment melanopsin and the cells in which melanopsin is expressed, the intrinsically photosensitive retinal ganglion cells (ipRGCs). The ipRGCs project broadly throughout the brain. Many of these projections terminate in areas known to mediate NIF effects, while others terminate in regions whose link to photoreception remains to be established. Additionally, the presence of ipRGC projections to areas of the brain with no known link to photoreception suggests the existence of additional ipRGC-mediated NIF effects. This review summarizes the known NIF effects of light and the role of melanopsin and ipRGCs in driving these effects, with an eye toward stimulating further investigation of the many and varied effects of light on physiology and behavior.

Keywords: amygdala, bed nucleus of the stria terminalis, melanopsin, opsin, optic nerve, retina

\section{Image-forming and nonimage-forming photoreception}

Light is a pervasive feature of the environment and the driver of an incredibly diverse collection of biological outputs, from basal physiological functions to higher order cognitive processes. Perhaps the most obvious biological adaptation to light is vision. Sighted animals use light to form a spatiotemporal representation of their environment, or colloquially, to "see". By detecting light that is reflected and emitted from objects and organisms in the environment, sighted animals can form images of their surroundings and determine the size, shape, color, and direction of motion of those objects and individuals. This process of "image formation" requires minimal spatial acuity to discern the boundaries between an object and the background space.

In addition to its role in vision, light also acts as a diffuse stimulus to exert many effects on behavior and physiology. These nonimage-forming (NIF) effects of light do not require the fine spatial acuity necessary for image formation. Rather, NIF effects are driven by changes in the general ambient light intensity, often in a wavelength- 
dependent manner. NIF effects of light are varied, ranging from regulation of basal physiology, such as heart rate and pupil diameter, to modulation of more complex behavioral phenomena, such as the entrainment of circadian rhythms and modulation of locomotor activity, to even higherorder cognitive processes, including alertness, anxiety, and mood. ${ }^{1-3}$

\section{The nonimage-forming effects of light}

A familiar and readily appreciable NIF effect of light is the modulation of pupil diameter. At dim light intensities, the pupil is dilated, maximizing the amount of light entering the eye. As irradiance increases, the pupil constricts until a minimal pupil area is attained. ${ }^{4}$ Constriction and dilation serve to maintain an optimal amount of light entering the eye under varying light conditions. Less apparent, but still readily measurable, is the effect of light on heart rate and core body temperature. In humans, nighttime light exposure causes an increase in both heart rate and core body temperature, relative to levels seen in darkness. ${ }^{5}$ Furthermore, light also modulates circulating hormone levels, notably melatonin and corticosterone. These effects are often gated depending on the time of day. For example, bright light at night induces suppression of circulating melatonin in humans within 10 to 20 min of light exposure. ${ }^{6}$ In contrast, suppression of plasma cortisol levels in humans is achieved by bright light during the subjective daytime. ${ }^{7}$

The NIF effects of light often have the same sign in nocturnal animals as in diurnal humans, although this is not always the case. For example, light causes the pupil to constrict in mice, ${ }^{4}$ and nighttime light suppresses plasma melatonin concentrations in CBA mice, ${ }^{8}$ just as it does in humans. ${ }^{6}$ However, light induces an increase in plasma corticosterone concentrations in Sprague-Dawley rats ${ }^{9}$ and C57BL/6J mice, ${ }^{10}$ opposite to the suppressive effect of bright light on cortisol in humans. ${ }^{7}$ Despite the opposite direction of the effect, light clearly modulates plasma hormone concentrations in both species, suggesting some degree of conservation in the pathways by which light modulates endocrine function.

In addition to modulating basal physiology, light also regulates more complex behavioral outputs. For example, light is the primary Zeitgeber, or "time giver", for the entrainment of circadian rhythms in both nocturnal and diurnal animals as well as other organisms. ${ }^{11}$ Tightly linked to the entraining effect of light is light's capacity to induce circadian phase shifts. In the absence of external cues, an organism must rely solely on its internal biological clock for time information. Any process governed by the circadian timing system will therefore not be linked to external time, and will "free run" with a period equal to that of the organism's intrinsic circadian rhythm. ${ }^{11}$ Under these free-running, constant conditions, a single light pulse is sufficient to induce a shift in the phase of the circadian timing system. ${ }^{12}$ The direction of this shift is gated, with light in the early subjective night generally causing phase delays and light in the late subjective night causing phase advances. ${ }^{13}$

Similar to the NIF effects of light on basal physiology, the effects of light on behavior may differ in diurnal versus nocturnal animals. For example, light acutely modulates the intensity of locomotor activity, even in the presence of an entraining light cycle, but the direction of the effect differs by photo-temporal niche. For example, in nocturnal animals, locomotor activity is acutely suppressed during a nighttime light pulse, a phenomenon known as "negative masking", while activity is acutely enhanced during a daytime dark pulse (positive masking). ${ }^{14}$ Conversely, acute light exposure in diurnal animals causes an increase in locomotor activity, while acute darkness suppresses locomotor activity. ${ }^{14}$ Light also acutely modulates sleep-wake propensity in different directions in nocturnal versus diurnal animals. Light exposure during either the daytime or nighttime decreases subjective sleepiness in humans as measured using the Karolinska Sleepiness Scale. ${ }^{5}$ Like many NIF responses, this effect is exaggerated following exposure to blue light. ${ }^{15}$ Nighttime blue light exposure also increases electroencephalogram power density, a more objective measure of sleep/wake status. ${ }^{16}$ In contrast to humans, light acutely induces sleep in nocturnal rodents. ${ }^{17,18}$ Notably, this effect is disrupted in mice lacking a photopigment that is particularly sensitive to blue light, ${ }^{17,18}$ suggesting commonalities in the mechanisms underlying the light-dependent modulation of sleep-wake propensity in diurnal and nocturnal animals, despite the alternate ultimate effects.

Light modulates higher-order cognitive processes, including alertness, anxiety, mood, and performance on a variety of cognitive tasks. ${ }^{3,19}$ Light increases subjective alertness as well, as measured by the Mental Effort Rating Scale. ${ }^{20}$ Light also impacts emotion, with potentially profound consequences for human psychological well-being. Light modulates anxiety, for instance, an effect that can be manifested in the lab as a modulation of the acoustic startle reflex (ACR). In nocturnal rodents, light enhances the ACR. ${ }^{19}$ In diurnal humans, darkness rather than light, enhances the startle reflex. ${ }^{21}$ Light also modulates responses 
to learned fear cues. Light enhances the freezing response of $\mathrm{C} 57 \mathrm{BL} / 6 \mathrm{~J}$ mice to a fear-conditioned tone, and this effect is independent of image-based contextual learning. ${ }^{22}$

These results have potential clinical implications for the many sufferers of anxiety disorders worldwide. In the United States alone, anxiety disorders afflict an estimated 40 million individuals over the age of 18 , a hallmark of which is the dysregulation of fear. ${ }^{23}$ Our observation that light modulates learned fear responses suggests that lighting conditions during therapy may affect patient outcome. This could have particular relevance for individuals with posttraumatic stress disorder (PTSD), which is analogous in many ways to fear conditioning. ${ }^{24}$ Incidence of PTSD in the US military in particular has been on the rise over the past decade, due to extensive, extended military commitments. ${ }^{25}$ Insight into the causes of PTSD, and the factors that exacerbate or alleviate its symptoms, would be timely and welcome. Light, by itself, already has therapeutic value in another affective disorder, seasonal affective disorder (SAD). Light therapy is the primary intervention for $\mathrm{SAD}$, and has been proposed as a treatment for "non-seasonal depression, bipolar depression, chronic depressive disorder, ante- and post-partum depression, late luteal phase dysphoric disorder, circadian sleep disorders, jet lag, shift work problems, and behavioral disturbance and insomnia in organic dementia". ${ }^{26}$ Given our results and the similarity of fear conditioning to PTSD, light could potentially be an effective component of treatment for this disorder as well. Secondly, our observation of the lightdependent enhancement of learned fear is, to our knowledge, the first observation of an effect of light on a learned behavioral response in mammals. It is certainly within reason that lighting conditions could affect performance in other learning and memory paradigms. For example, light modulates performance in digit recall, serial addition-subtraction, logical reasoning tasks, letter cancellation tasks, and reaction time tasks. ${ }^{3}$ In conjunction with our results, these observations support the prediction that light may enhance declarative memory performance in other tasks.

All of the effects of light described above are either demonstrated or presumed to be NIF. This interpretation is supported by several lines of evidence. First, these responses are dose-dependent; ie, they are directly correlated with the ambient light intensity across ranges where vision is already fully competent. Second, they generally persist in the absence of rod and cone cells, which are required for image formation. This persistence is due to intrinsically photosensitive retinal ganglion cells (ipRGCs), a third class of retinal photoreceptor that can mediate NIF effects. Third, many NIF effects of light are wavelength-dependent. If image formation were the causative agent of these effects of light, the likely prediction would be that the peak spectral sensitivity of NIF responses would coincide with the peak spectral sensitivity of photopic vision ( $555 \mathrm{~nm}) .{ }^{27}$ However, the majority of NIF responses are particularly sensitive to blue light stimulation ( $480 \mathrm{~nm})$. The blue-light sensitivity of sleep has been previously mentioned. In addition, suppression of melatonin, ${ }^{28}$ pupil constriction, ${ }^{29}$ and circadian phase shifting, ${ }^{30}$ are all preferentially sensitive to blue light. Even the effects of light on cognitive function are particularly sensitive to shorter wavelengths of light. ${ }^{16,20,31}$

\section{Melanopsin, ipRGCs, and NIF responses}

All responses to light in animals are, at the outset, instigated by the detection of photons by sensory neurons. In mammals, the exclusive sensory interface for all photoresponses is the retina. The outermost layer of the retina is populated by the photoreceptive rods and cones, which are the primary photoreceptors for image-forming vision. Rod/cone synaptic output is modulated by horizontal cells, and transduced via bipolar and amacrine cells to retinal ganglion cells (RGCs), which form the innermost layer of the retina. The axons of the RGCs exit the retina to form the optic nerve, ultimately to terminate throughout the brain. About 3\%-4\% of the RGCs are also photosensitive, ${ }^{32}$ thereby earning the name "intrinsically photosensitive RGCs" (ipRGCs). ${ }^{33,34}$ By virtue of their dual classification as retinal ganglion cells and their intrinsic photosensitivity, ipRGCs can convey information from the rods and cones, just like conventional RGCs, or they can initiate their own, independent photoresponses. ${ }^{35}$ Under normal polychromatic lighting conditions, they likely do both simultaneously. ipRGCs owe their photosensitivity to expression of the light-sensitive protein melanopsin (Gene symbol Opn4). ${ }^{33,36}$ Like rod and cone opsins, melanopsin is a $\mathrm{G}$ protein-coupled receptor with a covalently linked, photolabile chromophore. Activation of melanopsin initiates an intracellular phosphoinositide signaling cascade leading to neuronal depolarization and the generation of action potentials; this cascade is quite distinct from the cyclic nucleotide-based cascade that is activated by the rod and cone opsins. ${ }^{37}$ The evidence demonstrating that melanopsin is, indeed, the photopigment of the ipRGCs has been summarized elsewhere. ${ }^{35,37}$ The focus here is on the role of melanopsin and the ipRGCs in NIF responses.

As noted above, the rods and cones are the photoreceptors primarily responsible for image-forming vision. 
The rods and cones are also capable of driving NIF responses at low to medium light intensities, however, animals lacking functional rods and cones retain normal NIF photoresponses at all but the lowest light intensities., ${ }^{1,2}$ Extensive inquiry has revealed that this residual NIF capability is mediated by the ipRGCs. The sufficiency of the ipRGCs for driving NIF responses has been demonstrated by various methods. For example, animals lacking rod and cone input due to genetic mutation, retinal degeneration, or synaptic blockade retain the capacity to constrict their pupils in response to light, ${ }^{38}$ to suppress melatonin in response to light, ${ }^{39}$ to suppress their locomotor activity during light exposure, ${ }^{14}$ and to undergo a phase shift in locomotor activity rhythm in response to a nighttime light pulse. ${ }^{40}$ Animals lacking rods, cones, and melanopsin altogether, however, completely lack these NIF photoresponses. ${ }^{30,41}$ This absence of NIF capability demonstrates that the rods, cones, and ipRGCs together constitute the complete mammalian photosensory repertoire, and that melanopsin-bearing ipRGCs are therefore the sole drivers of NIF responses in the absence of rods and cones. It should be noted that melanopsin expression has also been observed in the mammalian iris, and this iris-resident melanopsin is capable of partially driving pupil constriction under very high light intensities. ${ }^{42}$

Melanopsin-based photoreception is sufficient, at middling light intensities, to drive a range of NIF responses. However, as noted, the rods and cones are also capable of driving pupil constriction, masking, phase shifting, and circadian entrainment at these middling light intensities in the absence of melanopsin, suggesting some level of redundancy. ${ }^{4,43}$ Yet the contributions of the $\mathrm{rod} /$ cone and melanopsin/ipRGC systems differ at extremely low- and high-light intensities. At low irradiances, the rods and cones are required for the normal expression of NIF responses, while at high irradiances melanopsin is required for full expression of the response. ${ }^{4,43}$ Furthermore, the ipRGCs themselves constitute a necessary component of the NIF pathway, apart from their intrinsic photosensitivity. The NIF capacity of the rods and cones is severely attenuated if the ipRGCs are selectively ablated, demonstrating that although the rods and cones can drive NIF responses by themselves, the ipRGCs are the primary conduit for rod-cone information to NIF centers in the brain. ${ }^{44-46}$

Melanopsin and the ipRGCs are therefore a critical component of the cascades leading to light-driven pupil constriction, circadian entrainment, circadian phase shifting, negative masking, and sleep induction. As described previously, the NIF effects of light extend far beyond these outputs, from basal physiological responses such as hormone secretion, to higher order functions such as modulation of alertness and emotion. Although direct evidence linking melanopsin and the ipRGCs to these effects is limited, circumstantial evidence supports a role for this system in the generation of these NIF effects. This evidence is based in part on the recognition that many NIF responses are most sensitive to light in the blue-wavelength region of the spectrum, which coincides with the peak sensitivity of melanopsin. ${ }^{37}$ Some of these blue-light sensitive NIF responses are known to be linked to melanopsin function, including pupil constriction, ${ }^{4,30,41,47}$ phase shifting, ${ }^{43,47}$ and sleep induction. ${ }^{16}$ Others have as yet not been definitively linked to melanopsin and the ipRGCs, such as heart rate and body temperature regulation, ${ }^{15}$ and cognitive processes including alertness, ${ }^{20}$ and emotional processing. ${ }^{48}$ Given their blue light sensitivity, it is likely that melanopsin and the ipRGCs play a role in these responses as well. It is also of note that a missense variant in the melanopsin gene is associated with SAD, further supporting a role for melanopsin in the light-dependent modulation of emotion. ${ }^{49}$ In addition to the fact that these effects are most efficiently elicited by blue light, anatomical evidence supports a role for melanopsin and the ipRGCs in these and other NIF responses. The ipRGCs project broadly to regions throughout the brain, many of which are known to mediate some of the aforementioned NIF responses. ${ }^{50,51}$ For example, the ipRGCs are the dominant direct retinal projection to the suprachiasmatic nuclei, which mediate the effects of light on circadian entrainment and circadian phase shifting. The ipRGCs also send projections to the olivary pretectal nucleus, which is involved in the pupillary light reflex. Provocatively, the ipRGCs project to regions of the brain known to be involved in NIF responses, but whose links to photoreception have not been definitively determined. The ipRGC projection to the bed nucleus of the stria terminalis $(\mathrm{BNST})^{51}$ may play a role in the manifestation of the effect of light on anxiety. As mentioned earlier, light modulates anxiety, as demonstrated by light-dependent changes in the acoustic startle reflex. This effect is strongly associated with activity in the BNST. ${ }^{52}$ Similarly, ipRGCs also terminate in the medial amygdala (MEA), ${ }^{51}$ which is activated during stress responses. ${ }^{53}$ Like the BNST, the MEA is a component of the extended amygdala. ${ }^{52}$ Interestingly, lesions of the MEA alter light-enhanced startle and open-field behavior, as well as other behavioral measures of anxiety and stress. ${ }^{54}$ Along with ipRGC-BNST connections, ipRGC-MEA connections may mediate some of the effects of light on anxiety. The ipRGCs have been suggested to mediate the exacerbating effect 
of light on migraine headaches, a hypothesis based in part on anatomical evidence. ${ }^{55}$ Taken together, there is strong circumstantial evidence of a role for melanopsin, ipRGCs, and the NIF visual system in human health and disease. ${ }^{56}$

Finally, additional complexity in melanopsin/ipRGC form and function is conferred by the existence of multiple ipRGC subtypes. Although we have thus far discussed ipRGCs as a monolithic entity, there is, in fact, significant diversity among ipRGCs. ipRGC subtypes differ in morphological properties, including retinal stratification, molecular markers, melanopsin expression, and dendritic field size and ramification, as well as in functional properties, including light-evoked electrophysiology, and terminal projections. ${ }^{2}$ This heterogeneity likely underlies specific roles for various ipRGC subtypes in driving specific NIF responses.

Clearly, light regulates and modulates behavior and physiology in numerous ways, at all levels of biological organization, with potentially profound consequences for an organism's health and well-being. The NIF actions of light allow an organism to adapt to changing environmental conditions and endure future challenges by biasing responses toward the most favorable outcome for survival, ie, changing pupil diameter allows optimal vision, entrainment of circadian rhythms allows anticipation of environmental conditions and resource availability, and heightened anxiety primes an organism for appropriate defensive responses when they are most vulnerable. Melanopsin and melanopsin-expressing ipRGCs have a critical role in the normal expression of many of these NIF responses, and they likely contribute to the manifestation of most NIF responses. Furthermore, the broad distribution of ipRGC projections raises the tantalizing possibility that additional, novel NIF effects of light remain to be identified. Additional studies should be performed with the aim of elucidating and characterizing the NIF effects of light on behavior and physiology and their implications for human health.

\section{Disclosure}

The authors report no conflicts of interest in this work.

\section{References}

1. Hatori M, Panda S. The emerging roles of melanopsin in behavioral adaptation to light. Trends Mol Med. 2010;16(10):435-446.

2. Schmidt TM, Chen SK, Hattar S. Intrinsically photosensitive retinal ganglion cells: many subtypes, diverse functions. Trends Neurosci. 2011;34(11):572-580.

3. Vandewalle G, Maquet P, Dijk DJ. Light as a modulator of cognitive brain function. Trends Cogn Sci. 2009;13(10):429-438.

4. Lucas RJ, Hattar S, Takao M, Berson DM, Foster RG, Yau KW. Diminished pupillary light reflex at high irradiances in melanopsinknockout mice. Science. 2003;299(5604):245-247.
5. Ruger M, Gordijn MC, Beersma DG, de Vries B, Daan S. Time-of-daydependent effects of bright light exposure on human psychophysiology: comparison of daytime and nighttime exposure. Am J Physiol Regul Integr Comp Physiol. 2006;290(5):R1413-R1420.

6. Lewy AJ, Wehr TA, Goodwin FK, Newsome DA, Markey SP. Light suppresses melatonin secretion in humans. Science. 1980;210(4475): 1267-1269.

7. Jung CM, Khalsa SB, Scheer FA, et al. Acute effects of bright light exposure on cortisol levels. J Biol Rhythms. 2010;25(3):208-216.

8. Kennaway DJ, Voultsios A, Varcoe TJ, Moyer RW. Melatonin and activity rhythm responses to light pulses in mice with the Clock mutation. Am J Physiol Regul Integr Comp Physiol. 2003;284(5): R1231-R1240.

9. Mohawk JA, Pargament JM, Lee TM. Circadian dependence of corticosterone release to light exposure in the rat. Physiol Behav. 2007;92(5):800-806.

10. Ishida A, Mutoh T, Ueyama T, et al. Light activates the adrenal gland: timing of gene expression and glucocorticoid release. Cell Metab. 2005;2(5):297-307.

11. Herzog ED. Neurons and networks in daily rhythms. Nat Rev Neurosci. 2007;8(10):790-802.

12. Takahashi JS, DeCoursey PJ, Bauman L, Menaker M. Spectral sensitivity of a novel photoreceptive system mediating entrainment of mammalian circadian rhythms. Nature. 1984;308(5955):186-188.

13. Nelson DE, Takahashi JS. Sensitivity and integration in a visual pathway for circadian entrainment in the hamster (Mesocricetus auratus). J Physiol. 1991;439:115-145.

14. Mrosovsky N. Masking: history, definitions, and measurement. Chronobiol Int. 1999;16(4):415-429.

15. Cajochen C, Münch M, Kobialka S, et al. High sensitivity of human melatonin, alertness, thermoregulation, and heart rate to short wavelength light. J Clin Endocrinol Metab. 2005;90(3):1311-1316.

16. Lockley SW, Evans EE, Scheer FA, Brainard GC, Czeisler CA, Aeschbach D. Short-wavelength sensitivity for the direct effects of light on alertness, vigilance, and the waking electroencephalogram in humans. Sleep. 2006;29(2):161-168.

17. Altimus CM, Güler AD, Villa KL, McNeill DS, Legates TA, Hattar S. Rods-cones and melanopsin detect light and dark to modulate sleep independent of image formation. Proc Natl Acad Sci U S A. 2008;105(50): 19998-20003.

18. Lupi D, Oster H, Thompson S, Foster RG. The acute light-induction of sleep is mediated by OPN4-based photoreception. Nat Neurosci. 2008;11(9):1068-1073.

19. Walker DL, Davis M. Anxiogenic effects of high illumination levels assessed with the acoustic startle response in rats. Biol Psychiatry. 1997;42(6):461-471.

20. Chellappa SL, Steiner R, Blattner P, Oelhafen P, Gotz T, Cajochen C. Non-visual effects of light on melatonin, alertness and cognitive performance: can blue-enriched light keep us alert? PLoS One. 2011; 6(1):e16429.

21. Grillon C, Pellowski M, Merikangas KR, Davis M. Darkness facilitates the acoustic startle reflex in humans. Biol Psychiatry. 1997;42(6): 453-460.

22. Warthen DM, Wiltgen BJ, Provencio I. Light enhances learned fear. Proc Natl Acad Sci US A. August 16, 2011;108(33):13788-13793.

23. Kessler RC, Chiu WT, Demler O, Merikangas KR, Walters EE. Prevalence, severity, and comorbidity of 12-month DSM-IV disorders in the National Comorbidity Survey Replication. Arch Gen Psychiatry. 2005;62(6):617-627.

24. Jovanovic T, Ressler KJ. How the neurocircuitry and genetics of fear inhibition may inform our understanding of PTSD. Am J Psychiatry. 2010;167(6):648-662.

25. Hermes ED, Rosenheck RA, Desai R, Fontana AF. Recent trends in the treatment of posttraumatic stress disorder and other mental disorders in the VHA. Psychiatr Serv. 2012;63(5):471-476.

26. Prasko J. Bright light therapy. Neuro Endocrinol Lett. 2008;29 Suppl 1: 33-64. 
27. Wald G. Human vision and the spectrum. Science. 1945;101(2635): 653-658.

28. West KE, Jablonski MR, Warfield B, et al. Blue light from light-emitting diodes elicits a dose-dependent suppression of melatonin in humans. J Appl Physiol. 2011;110(3):619-626.

29. Gamlin PD, McDougal DH, Pokorny J, Smith VC, Yau KW, Dacey DM. Human and macaque pupil responses driven by melanopsin-containing retinal ganglion cells. Vision Res. 2007;47(7):946-954.

30. Hattar S, Lucas RJ, Mrosovsky N, et al. Melanopsin and rod-cone photoreceptive systems account for all major accessory visual functions in mice. Nature. 2003;424(6944):76-81.

31. Vandewalle G, Gais S, Schabus M, et al. Wavelength-dependent modulation of brain responses to a working memory task by daytime light exposure. Cereb Cortex. 2007;17(12):2788-2795.

32. Ecker JL, Dumitrescu ON, Wong KY, et al. Melanopsin-expressing retinal ganglion-cell photoreceptors: cellular diversity and role in pattern vision. Neuron. 2010;67(1):49-60.

33. Berson DM, Dunn FA, Takao M. Phototransduction by retinal ganglion cells that set the circadian clock. Science. 2002;295(5557):1070-1073.

34. Hattar S, Liao HW, Takao M, Berson DM, Yau KW. Melanopsincontaining retinal ganglion cells: architecture, projections, and intrinsic photosensitivity. Science. 2002;295(5557):1065-1070.

35. Provencio I. Melanopsin Cells. The Senses: A Comprehensive Reference. New York, NY: Academic Press; 2008:423-431.

36. Provencio I, Rodriguez IR, Jiang G, Hayes WP, Moreira EF, Rollag MD. A novel human opsin in the inner retina. J Neurosci. 2000;20(2): 600-605.

37. Provencio I, Warthen DM. Melanopsin, the photopigment of intrinsically photosensitive retinal ganglion cells. WIRES Membr Transp Signal. 2012;1(2):228-237.

38. Lucas RJ, Douglas RH, Foster RG. Characterization of an ocular photopigment capable of driving pupillary constriction in mice. Nat Neurosci. 2001;4(6):621-626.

39. Lucas RJ, Freedman MS, Muñoz M, Garcia-Fernández JM, Foster RG. Regulation of the mammalian pineal by non-rod, non-cone, ocular photoreceptors. Science. 1999;284(5413):505-507.

40. Freedman MS, Lucas RJ, Soni B, et al. Regulation of mammalian circadian behavior by non-rod, non-cone, ocular photoreceptors. Science. 1999;284(5413):502-504.

41. Panda S, Provencio I, Tu DC, et al. Melanopsin is required for nonimage-forming photic responses in blind mice. Science. 2003;301(5632): $525-527$.

42. Xue T, Do MT, Riccio A, et al. Melanopsin signalling in mammalian iris and retina. Nature. 2011;479(7371):67-73.
43. Panda S, Sato TK, Castrucci AM, et al. Melanopsin (Opn4) requirement for normal light-induced circadian phase shifting. Science. 2002;298(5601):2213-2216.

44. Göz D, Studholme K, Lappi DA, Rollag MD, Provencio I, Morin LP. Targeted destruction of photosensitive retinal ganglion cells with a saporin conjugate alters the effects of light on mouse circadian rhythms. PLoS One. 2008;3(9):e3153.

45. Güler AD, Ecker JL, Lall GS, et al. Melanopsin cells are the principal conduits for rod-cone input to non-image-forming vision. Nature. 2008;453(7191):102-105.

46. Hatori M, Le H, Vollmers C, et al. Inducible ablation of melanopsinexpressing retinal ganglion cells reveals their central role in non-image forming visual responses. PLoS One. 2008;3(6):e2451.

47. Mure LS, Rieux C, Hattar S, Cooper HM. Melanopsin-dependent nonvisual responses: evidence for photopigment bistability in vivo. J Biol Rhythms. 2007;22(5):411-424.

48. Vandewalle G, Schwartz S, Grandjean D, et al. Spectral quality of light modulates emotional brain responses in humans. Proc Natl Acad Sci US A. 2010;107(45):19549-19554.

49. Roecklein KA, Rohan KJ, Duncan WC, et al. A missense variant (P10L) of the melanopsin (OPN4) gene in seasonal affective disorder. J Affect Disord. 2009;114(1-3):279-285.

50. Dacey DM, Liao HW, Peterson BB, et al. Melanopsin-expressing ganglion cells in primate retina signal colour and irradiance and project to the LGN. Nature. 2005;433(7027):749-754.

51. Hattar S, Kumar M, Park A, et al. Central projections of melanopsinexpressing retinal ganglion cells in the mouse. J Comp Neurol. 2006; 497(3):326-349.

52. Davis M, Walker DL, Miles L, Grillon C. Phasic vs sustained fear in rats and humans: role of the extended amygdala in fear vs anxiety. Neuropsychopharmacology. 2010;35(1):105-135.

53. Davern PJ, Head GA. Role of the medial amygdala in mediating responses to aversive stimuli leading to hypertension. Clin Exp Pharmacol Physiol. 2011;38(2):136-143.

54. Vinkers CH, Bijlsma EY, Houtepen LC, et al. Medial amygdala lesions differentially influence stress responsivity and sensorimotor gating in rats. Physiol Behav. 2010;99(3):395-401.

55. Noseda R, Kainz V, Jakubowski M, et al. A neural mechanism for exacerbation of headache by light. Nat Neurosci. 2010;13(2):239-245.

56. La Morgia C, Ross-Cisneros FN, Hannibal J, Montagna P, Sadun AA, Carelli V. Melanopsin-expressing retinal ganglion cells: implications for human diseases. Vision Res. 2011;51(2):296-302.
Eye and Brain

\section{Publish your work in this journal}

Eye and Brain is an international, peer-reviewed, open access journal focusing on clinical and experimental research in the field of neuro-ophthalmology. All aspects of patient care are addressed within the journal as well as basic research. Papers covering original research, basic science, clinical and epidemiological studies, reviews and

\section{Dovepress}

evaluations, guidelines, expert opinion and commentary, case reports and extended reports are welcome. The manuscript management system is completely online and includes a very quick and fair peer-review system, which is all easy to use. Visit http://www.dovepress.com/ testimonials.php to read real quotes from published authors. 